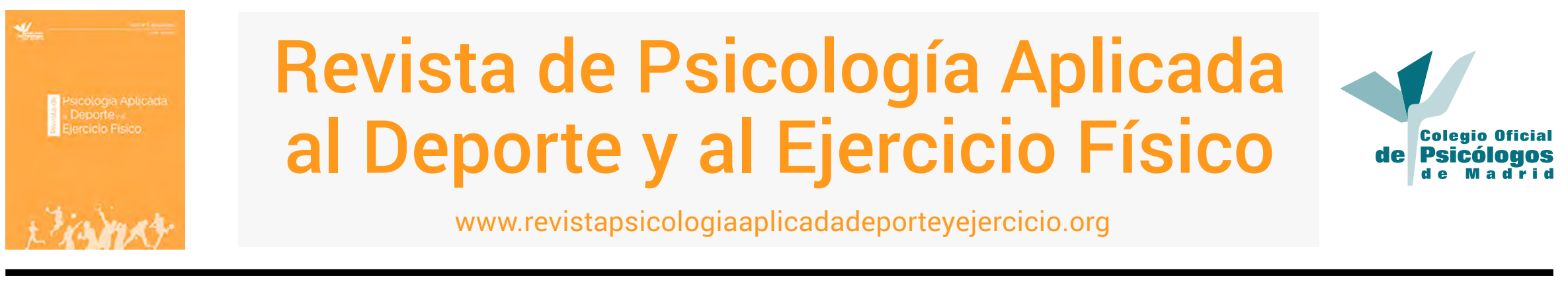

\title{
Sobreentrenamiento y deporte desde una perspectiva psicológica: estado de la cuestión
}

\author{
Fátima Sánchez-Beleña ${ }^{1}$ y Alejo García-Naveira Vaamonde ${ }^{2}$ \\ ${ }^{1}$ Conservatorio Superior de Danza de Madrid “María de Ávila”, España, ${ }^{2}$ Colegio Oficial de Psicólogos de Madrid , España
}

RESUMEN: La actividad física moderada presenta innumerables beneficios para la salud física y mental, pero a medida que se aumenta la cantidad de tiempo de dedicación y las cargas de trabajo durante los entrenamientos, puede provocar alguna sintomatología o problemas de salud, entre ellos, diferentes estados de fatiga que pueden desembocar en el Síndrome de Sobreentrenamiento (SSE). Los objetivos del presente artículo son establecer una distinción conceptual entre el SSE y otros conceptos relacionados, analizar cómo se evalúa para su detección y revisar estudios representativos sobre la temática publicados entre los años 2010 y 2017 . Se concluye que la sintomatología relacionada con el SSE es muy variada (cambios orgánicos, psicológicos, conductuales, etc.), puede presentar diferencias en función de la modalidad deportiva (mayor en deportes individuales), nivel de competición (mayor en alto rendimiento) e incluso de una persona a otra. Se suele detectar cuando hay síntomas evidentes del mismo y está avanzado, como el descenso del rendimiento deportivo, aunque su inicio es anterior. Por ello, se requiere un estudio individualizado del SSE, en el que se recomienda una detección temprana y un seguimiento periódico del mismo, siendo el POMS uno de los instrumentos más utilizados y eficaces debido a la variación del estado de ánimo relacionado con el síndrome. Además, a nivel práctico, es importante revisar y ajustar los tiempos de dedicación, cargas de entrenamiento y recuperación entre sesiones del deportista, así como el descanso, el sueño y la alimentación. El psicólogo del deporte puede asesorar y orientar a los técnicos deportivos sobre el SSE, evaluar e intervenir psicológicamente para su prevención y tratamiento.

PALABRAS CLAVES: Sobreentrenamiento, sobrecarga, fatiga y estado de ánimo.

\section{Overtraining and sports from a psychological perspective: the state of the question}

ABSTRACT: Moderate physical activity has innumerable benefits for physical and mental health, but as the amount of time of dedication and workloads during training increases, it may cause some symptoms or health problems, including different states offatigue that may lead to an overtraining syndrome (OS). This study was intended to establish a conceptual distinction between OS and other related concepts, to analyze how it is detected, and to review representative studies on the subject published between 2010 and 2017 . It is concluded that symptoms related to OS are very diverse (organic, psychological, behavioral changes, etc.), and may differ depending on the sport (more marked symptoms in individual sports), competition level (higher in high performance) and from one person to another. OS is usually detected when it is in an advanced stage and there are obvious symptoms, such as a decline in athletic performance, but its onset is earlier. An individualized study of OS is therefore required in which early detection and regular follow-up are recommended. POMS is one of the most commonly used and effective instruments because mood changes related to the syndrome. In addition, on a practical level, it is important to review and adjust the dedication times, training loads, and recovery time between sessions of the athlete, as well as rest, sleep, and diet. The sports psychologist may counsel and guide sports technicians on OS, evaluate, and apply psychological interventions for its prevention and treatment.

KEYWORDS: Overtraining, overload, fatigue, mood.

\footnotetext{
Fátima Sánchez es Psicóloga del Conservatorio Superior de Danza de Madrid "María de Ávila y Alejo García-Naveira es Psicólogo Coordinador de la Sección de Psicología de la Actividad Física y el Deporte del Colegio Oficial de Psicólogos de Madrid.
} 


\section{Overtraining e esporte sob uma perspectiva psicológica: estado em questão}

RESUMO: A atividade física moderada tem inúmeros benefícios para a saúde física e mental, mas à medida que a quantidade de tempo de dedicação e carga de trabalho durante o treinamento aumentam, pode causar alguns sintomas ou problemas de saúde, incluindo diferentes estados de fadiga que podem levar à Síndrome do Overtraining (SSE). Os objetivos deste artigo são estabelecer uma distinção conceitual entre a SSE e outros conceitos relacionados, analisar como é avaliada para detecção e analisar estudos representativos sobre o tema publicado entre 2010 e 2017. Conclui-se que os sintomas relacionados com a SSE são muito variados (mudanças orgânicas, psicológicas, comportamentais, etc.) e podem apresentar diferenças dependendo da modalidade esportiva (maior em esportes individuais), nível de competição (maior em alto desempenho) e até mesmo de uma pessoa para outra. Geralmente, é detectada quando há sintomas óbvios e está avançada, como o declínio do desempenho atlético, embora seu início seja anterior. Portanto, é necessário um estudo individualizado da SSE, no qual é recomendada a detecção precoce e o acompanhamento periódico, sendo o POMS um dos instrumentos mais utilizados e efetivos devido à variação do humor relacionado com a síndrome. Além disso, a um nível prático, é importante rever e ajustar os tempos de dedicação, cargas de treinamento e recuperação entre as sessões do atleta, bem como o descanso, o sono e a alimentação. O psicólogo do esporte pode assessorar e orientar os técnicos esportivos sobre a SSE, avaliar e intervir psicologicamente para sua prevenção e tratamento.

PALAVRAS-CHAVE: Overtraining, sobrecarga, fadiga e humor.

Artículo recibido: 05/05/2017 | Artículo aceptado: 09/07/2017

El ejercicio físico puede provocar efectos tanto positivos como negativos en la persona en función de la "dosis" empleada en cuanto a intensidad del ejercicio, tiempo de dedicación, carga de trabajo y recuperación entre entrenamientos, entre otras cuestiones, así como aspectos externos de la actividad que influye en la persona como son los hábitos de descanso, sueño, ocio y alimentación (García-Naveira y Locatelli, 2015; Nogueira, Salguero y Márquez, 2017). Estos autores indican que la práctica moderada de ejercicio físico se ha asociado a múltiples beneficios para la salud física y psicológica, como el bienestar, la calidad de vida, el estado de ánimo, reduciendo la depresión, disminuyendo la ansiedad, favoreciendo el afrontamiento del estrés e incrementando la autoestima, mejorando las relaciones sociales, etc., y también, previniendo algunos problemas físicos y de salud como la obesidad y los trastornos cardiovasculares.

Sin embargo, en deportistas de alto rendimiento, el incremento continuado de las cargas de trabajo y los tiempos de entrenamiento, así como la reducción de los tiempos de recuperación entre sesiones, puede estar asociado a algunos problemas de salud física y psicológica (Slivka, Hailes, Cuddy y Ruby, 2010). Uno de esos efectos colaterales del deporte sería el Síndrome de Sobreentrenamiento (SSE) y las distintas formas de fatiga extrema asociada a la actividad física intensa (Mora, 2006). Si no se realiza una intervención temprana, la sintomatología continúa aumentando su intensidad y diversificándose, pudiendo perjudicar el rendimiento deportivo y la salud de la persona, e incluso llegar al abandono de la actividad deportiva.

Dada la importancia del SSE en el deporte, los objetivos del presente trabajo son abordar la definición y delimitación conceptual del SSE frente a otros conceptos de fatiga extre- ma asociada a la actividad física intensa, los instrumentos de evaluación empleados para su detección y realizar una revisión de trabajos de investigación más representativos de los últimos 7 años sobre la temática (2010-2017).

\section{Definición y Delimitación Conceptual}

Existen varios conceptos que hacen referencia a los estados de fatiga extrema que pueden sufrir los deportistas como son la fatiga, sobrecarga funcional y no funcional, burnout y sobreentrenamiento (Sánchez-Beleña, 2011). A continuación se describe cada uno de ellos.

La fatiga hace referencia a la disminución de la capacidad funcional del organismo por un exceso de exigencia física y mental dentro del entrenamiento (Zintl, 1991). Sería la sensación de falta de energía, agotamiento o cansancio, que puede aparecer como consecuencia de cualquier sesión de entrenamiento y que se recupera fácilmente en unos días cuando cesa la actividad.

La sobrecarga funcional aparece cuando se incrementa la intensidad del entrenamiento durante un periodo corto de tiempo o se repite de forma prolongada un movimiento que hace que un músculo tenga que intervenir de forma específica, lo cual no impedirá seguir rindiendo o haciendo el gesto deportivo en cuestión, pero que poco a poco se irá alterando (Schmikli, Brink, De Vries, Backx, 2011). Se puede recuperar con relativa facilidad en un periodo de descanso, que puede variar desde algunos días hasta semanas. Por otro lado, la sobrecarga no funcional se refiere al estado de sobrecarga extrema provocado por el desequilibrio sostenido entre el organismo y la carga del entrenamiento o repetición del movi- 
miento, teniendo la necesidad de cesar la actividad o pudiendo llegar inclusive a la lesión deportiva. Aquí el periodo de recuperación necesario es más amplio, pudiendo alcanzar desde semanas a meses.

Otro problema que pueden presentar los deportistas es el burnout (estar quemado), que representa un estado de agotamiento físico, emocional y mental (Carlín y Garcés de los Fayos, 2010). Freudenberger (1974) fue el primer autor en utilizar este término y lo define como un cuadro amotivacional profundo que conduce a una pérdida de interés y de la asunción de responsabilidades. Mora (2006) expuso que existen algunas características de personalidad que funcionarían como factores de predisposición ante el burnout como son la sensibilidad a los sentimientos, necesidades de los demás, dedicación al trabajo, idealismo, elevada ansiedad y autoexigencia. La recuperación en los casos de burnout dura varios meses o incluso años (Lemyre, Roberts y Stray-Gundersen, 2007).

El sobreentrenamiento a corto plazo, también llamado saturación, se caracteriza por síntomas como fatiga física y mental, mal humor, apatía y trastornos del sueño entre otros. (González-Boto, Molinero y Márquez, 2006). En este momento, este estado aún es fácilmente reversible, interrumpiendo las sesiones de entrenamiento deportivo. Cuando existe un déficit de recuperación, el estado de saturación que definíamos anteriormente se cronifica y hablamos de sobreentrenamiento a largo plazo (Meeusen et al., 2006). Kellman lo define en el año 2002 como un síndrome que aparece cuando un entrenamiento intenso o demasiado prolongado excede la capacidad de recuperación del organismo. En este caso, interrumpir la actividad no es suficiente, es necesario intervenir sobre la sintomatología específica que manifiesta cada deportista. La recuperación total puede tardar meses o incluso años. Pueden aparecer alteraciones fisiológicas y psicológicas y síntomas desadaptativos (Meeusen et al., 2006). Se da una combinación compleja de síntomas físicos y psicológicos, que se presentarán con detalle en el siguiente apartado, y que adquiere la denominación de síndrome (Lemyre et al., 2007). Destacar que la diferencia fundamental con el burnout es que en el sobreentrenamiento la motivación se mantiene, mientras que en el burnout se da un cuadro amotivacional generalizado (Mora, 2006).

\section{Síntomas de Sobreentrenamiento: Descripción del Síndrome}

En un principio se atribuía el sobreentrenamiento a factores relacionados exclusivamente con la práctica deportiva. Hoy se aceptan como desencadenantes también las viven- cias de estrés y el efecto de varios estresores, no sólo los relacionados con el entrenamiento, sino también los efectos del descanso, la alimentación y la sobrecarga psicológica (Pancorbo, 2003; Prendas, Castro y Araya- Vargas, 2007).

Algunos autores (Meeusen et al. 2006; González-Boto et al., 2006) han explicado en parte el sobreentrenamiento haciendo referencia al Síndrome General de Adaptación descrito por Selye (1936). Al someter al organismo a una situación de estrés se alterarían las secreciones de adrenalina, noradrenalina y cortisol para poder modificar el aporte de glucosa y el gasto metabólico y así hacer frente al estresor. Si esta exposición al estímulo estresante se mantuviera en el tiempo sería necesario modificar los niveles de algunos neurotransmisores, lo que provocaría, con el tiempo, una afectación de la función hipotalámica y de otras respuestas neurales (Meeusen, 1999). En el momento en que se sobrepasa la capacidad de adaptación del organismo, el sujeto ya no es capaz de enfrentarse a nuevos esfuerzos. Entonces es cuando aparece la fatiga y el bajo rendimiento deportivo (Suay, Sanchís y Salvador, 1997). Tal y como describieron Meeusen et al. (2006), los deportistas serían capaces de comenzar sus sesiones de entrenamiento, pero serían incapaces de mantener o de incrementar el ritmo o el esfuerzo físico.

Anshel (2003) destacó los estresores más relevantes y específicos para el ámbito deportivo a corto plazo, como es el dolor asociado a una lesión, el temor ante los posibles errores técnicos, no estar en la forma física adecuada, no obtener resultados favorables, recibir comentarios negativos del público o de los compañeros, etc.. A largo plazo, otros factores estresantes serían la monotonía del entrenamiento, las demandas de la competición, la falta de apoyo social, la represión de las tensiones y los conflictos y el exceso de responsabilidad (Márquez, 2006).

Los síntomas del sobreentrenmiento son muy variados y abarcan desde la apatía, las dificultades para conciliar el sueño hasta la mayor vulnerabilidad para sufrir lesiones, incluyendo importantes modificaciones del sistema metabólico que tardarían varias semanas o algunos meses en restablecerse (Mora, 2006; Sánchez-Beleña, 2011). En la Tabla 1 , se presenta con mayor detenimiento los diferentes síntomas asociados al sobreentrenamiento.

Algunas investigaciones señalan que la sintomatología del sobreentrenamiento es levemente diferente para cada modalidad deportiva (p. ej., mayor en deportes individuales frente a los de equipo), pero en realidad los síntomas varían de una persona a otra (Stevenson, 2008). El único factor presente en todas las modalidades deportivas es la disminución del rendimiento (Saremi, 2009). Prueba de ello es que la Asociación Olímpica Británica se planteó en 1999 
Tabla 1. Resumen de síntomas de sobreentrenamiento

\begin{tabular}{|c|c|}
\hline Generales & $\begin{array}{l}\text { Cansancio, insomnio, pérdida de apetito, disminución de peso corporal, cefalea, dolores musculares, infecciones } \\
\text { frecuentes, trastornos digestivos, amenorrea acompañada de osteoporosis }\end{array}$ \\
\hline Psicológicos & $\begin{array}{l}\text { Depresión, ansiedad, descenso de la autoestima, apatía, disminución de la concentración, fatiga mental constante, } \\
\text { inestabilidad emocional, desinterés }\end{array}$ \\
\hline \multirow[t]{2}{*}{ Bioquímicas } & Aumenta la urea, el ácido úrico, el amoniaco basal, el cortisol, las catecolaminas, el potasio. \\
\hline & $\begin{array}{l}\text { Disminuye la testosterona, la hemoglobina, el hierro, la ferritina y los niveles de Zinc, Cobalto, Cobre, Alumino, Selenio, } \\
\text { Magnesio y Manganeso y las Vitaminas E y C. }\end{array}$ \\
\hline Hormonales & Aumento del cortisol y descenso de la testosterona. Alteraciones en las catecolaminas y la tiroxina \\
\hline Fisiológicos & Incremento de la frecuencia cardíaca y la tensión arterial y aumento del consumo de oxígeno \\
\hline Sistema músculo esquelético & $\begin{array}{l}\text { Aumenta la frecuencia de las lesiones por sobreuso, en especial insercionitis, tendinitis, periostitis, condromalacia y } \\
\text { fracturas por estrés }\end{array}$ \\
\hline Aspectos técnicos & $\begin{array}{l}\text { Perdida de la coordinación, disminución de la capacidad para hacer frente a una gran cantidad de información, repe- } \\
\text { tición de los errores que ya fueron superados anteriormente. Dificultades para alcanzar las metas propuestas }\end{array}$ \\
\hline
\end{tabular}

comenzar a denominar este estado de sobreentrenamiento como "Síndrome de bajo rendimiento inexplicable" (Budgett et al, 2000).

El sobreentrenamiento en el ámbito deportivo está relacionado con muchas lesiones músculo esqueléticas. La elevada frecuencia e intensidad de los entrenamientos aumenta la sobrecarga muscular y la probabilidad de lesiones por sobreuso o incluso fracturas por estrés (Yoke, 2006). Las variaciones del estado de ánimo implican cambios a nivel biológico, incluyendo inmunosupresión y la reducción de glucógeno disponible en el sistema muscular, de ahí la mayor vulnerabilidad ante las lesiones y el descenso en el rendimiento.

Dentro del ámbito deportivo, se focaliza en exceso en el descenso continuado del rendimiento (Andrade, Arce y Seoane, 2000). Sin embargo, no es el primer síntoma que aparece (Nicholls, McKenna, Polman y Backhouse, 2011). Cuando se manifiesta el descenso en el rendimiento, el deportista ya lleva tiempo expuesto a un exceso de demandas que su organismo no ha sido capaz de manejar. También hay algunos síntomas generales que son los primeros en aparecer, como por ejemplo las dificultades para dormir, el comportamiento irritable, disminución de la atención y la concentración durante el entrenamiento, menor control en las ejecuciones, entre otros. La dificultad estriba en que al ser síntomas tan variados y tan difusos, no se identifican como los orígenes del sobreentrenamiento (Mora, 2006; Sánchez-Beleña, 2011).

\section{Instrumentos de Medida}

Dentro de este apartado se revisan las diferentes formas de evaluar el sobreentrenamiento. Durante muchos años se ha diagnosticado a partir de indicadores exclusivamente biológicos como los niveles de cortisol en saliva o en sangre, los niveles de testosterona, citoquinasas (CK) y lactato (Steinacker et al, 2000; Steinacker, Lormes, Reissnecker y Liu, 2004; Meeusen et al., 2004). Como señalan estos autores, no obstante, monitorizar estas medidas requiere el uso de pruebas invasivas y análisis en laboratorio que implican un elevado coste económico, además los resultados obtenidos no han sido siempre consistentes.

Por ejemplo, recientemente, Cadegiani y Kater (2017) revisaron 38 estudios que utilizaban la mediciones de las variaciones hormonales como consecuencia del sobreentrenamiento y encontraron que la medida basal de la adenocorticotropa (ACTH), la hormona del crecimiento $(\mathrm{GH})$ y prolactina, no parecen jugar una herramienta precisa para el diagnóstico, pero sí hay alteraciones durante los periodos de mayor exigencia del entrenamiento. Aun así, se necesitan más estudios para mejorar la exactitud y los puntos de corte de los marcadores hormonales.

Como métodos menos invasivos para evaluar el sobreentrenamiento surgieron algunos autoinformes y posteriormente instrumentos estandarizados. Las primeras pruebas 
fueron las escalas subjetivas de fatiga y de esfuerzo físico percibido. Se diseñaron instrumentos como la escala Rate of Perceived Exertion (RPE; Borg, 1970) en la que se plantean preguntas del tipo "¿Cómo has percibido el esfuerzo que acabas de hacer? El deportista debe valorar el esfuerzo percibido en una escala tipo Likert de 6 a 20, donde 6 es "Esfuerzo Mínimo" y 20 es el "Esfuerzo Máximo". En este sentido, el mayor esfuerzo y fatiga percibida podrían ser unos indicadores de intensidad y carga de trabajo en el deporte, pudiendo además representar una sintomatología de sobreentrenamiento (Clemente, Muñoz, Ramos, Navarro y González-Ravé, 2009; Suay y Fernández de Castro, 2001).

Lemyre et al, (2007) crearon una prueba a partir de los síntomas de sobreentrenamiento más frecuentes. Dicha prueba denominada Short Overtraining Symptoms Questionnaire (SOSQ) consta de 5 preguntas en las que se debía valorar de 1 (mínimo) a 10 (máximo) en una escala tipo Likert cómo se había sentido la persona últimamente en cuanto a la calidad del sueño, el apetito, la energía, las ganas de entrenar y la calidad del entrenamiento.

El grupo de trabajo Surentraînement de la Sociedad Francesa de Medicina del Deporte (SFMD) ha desarrollado un instrumento denominado Cuestionario de Síntomas de Sobreentrenamiento (CSSE; Legros, 1993). La prueba recoge los síntomas más representativos del sobreentrenamiento. A partir de los síntomas clásicos que aparecen en la literatura (dificultades para mantener la atención, dificultades para conciliar el sueño, problemas de memoria, dolores de cabeza o descenso de la autoconfianza), los autores desarrollaron un cuestionario de 58 ítems, con una respuesta dicotómica donde debe señalarse "sí o no" según se haya padecido o no durante el último mes la sintomatología que muestra el ítem. A partir de 20 ítems señalados se considera que existe sobreentrenamiento (Maso, Lac y Brunn, 2005). El CSSE se considera un buen indicador de las señales tempranas del sobreentrenamiento (Suay, Ricarte y Salvador, 1998). En España contamos con la versión adaptada al español (Del Villar, Pegueros y Franco, 2008).

El Recovery Stress Questionnaire for Athletes (RESTQ-Sport) es un cuestionario desarrollado por Kellman y Kallus en 2001 y destinado a medir los cambios psicológicos como consecuencia del sobreentrenamiento. Consta de 76 ítems (28 específicos y 48 no específicos) distribuidos en 19 escalas, 12 de ellas generales (estrés general, estrés emocional, estrés social, conflictos/ presión, fatiga, falta de energía, alteraciones físicas, éxito, recuperación social, recuperación física, bienestar general y calidad del sueño) y 7 escalas específicas para deportistas (descanso alterado, fatiga emocional/ burnout, forma física/ lesiones, bienestar/ estar en forma, burnout/ realización personal, autoeficacia, autorre- gulación). Los sujetos deben indicar de forma retrospectiva en una escala Likert graduada de 1 (nuca) a 6 (siempre) puntos la frecuencia con que han participado en diferentes actividades durante los últimos 3 días/noches. La versión española denominada Cuestionario de Recuperación-Estrés par Deporistas ha sido desarrollada por González-Boto, Salguero, Tuero, Kellmann y Márquez (2008). Las escalas de esta prueba son sensibles a los entrenamientos que pueden estar asociados al desarrollo de sobreentrenamiento (Molinero, Salguero y Márquez, 2011).

El Profile Of Mood States (POMS; McNair, Lorr y Doppleman, 1971) diseñado inicialmente para el ámbito clínico, es considerado un instrumento importante en la Psicología del Deporte debido a la relación existente entre los estados de ánimo y el rendimiento deportivo (De la Vega, Galán, Ruiz-Barquín y Tejero, 2013; De la Vega, Ruiz-Barquín, Tejero y Rivera, 2014; LeUnes y Burger, 2000).

La versión original del POMS consta de 65 adjetivos (McNair et al., 1971) en los que se debe valorar en una escala tipo Likert el grado de identificación con cada uno de ellos, desde 0 (Nada) a 4 (Mucho). Los ítems se agrupan en las escalas de Tensión (refleja un aumento de la tensión músculo-esquelética), Depresión (refleja un bajo estado de ánimo o un estado de ánimo deprimido, aunque no se basa en los criterios clínicos para el diagnóstico de la depresión, tal y como indican Martin, Andersen y Gates, 2000), Cólera (muestra un estado de ánimo de enfado y antipatía ante los demás), Vigor (representa un estado de elevada energía), Fatiga (representa un estado de baja energía), Confusión (se caracteriza por la confusión mental y el desorden) y Amistad (refleja el nivel de rechazo hacia los demás).

EI POMS cuenta con numerosas versiones reducidas y adaptaciones al español (Andrade, Arce y Seoane, 2002; Andrade, España y Rodríguez, 2016), como por ejemplo, la versión de 29 items para deportistas adolescentes (Andrade, Arce, Garrido, Torrado y De Francisco, 2011) o la versión de 30 items para deportistas adultos y población general (Andrade, Arce, De Francisco, Torrado y Garrido, 2013). También existe una versión tridimensional de este cuestionario para adultos denominada POMS-VIC (De la Vega, Ruiz-Barquín, Borges y Tejero-González, 2014). Estos autores plantean que añadiendo las dimensiones de valencia y control junto con la intensidad que ya se medía en las versiones anteriores de la prueba, se puede tener una visión más completa de la experiencia anímica del deportista.

Además, de la relación con el rendimiento deportivo, el POMS se ha empleado para analizar el efecto de la participación en programas de ejercicio, así como en el seguimiento psicológico del sobreentrenamiento y la fatiga, debido a que los estados de ánimo fluctúan con relación a la intensidad 
del entrenamiento (Andrade et al., 2000; Bonete, Moya y Suay, 2009; Piacentini y Meeusen, 2014). Estos autores indican un aumento considerable de las puntuaciones en las escalas Fatiga y Depresión, así como una disminución notable en la escala Vigor al final de los períodos de entrenamiento más intenso, sintomatología asociada al sobreentrenamiento.

\section{Revisión de Investigaciones}

Se han revisado trabajos representativos de los últimos 7 años, relacionadas con los términos "sobrecarga" y "sobreentrenamiento" en el ámbito deportivo. Para ello, se han realizado búsquedas en las siguientes bases de datos: Psyclnfo, PsicoDoc, Web of Science y Scopus, desde el años 2010 hasta el mes de octubre de 2017. Como resultado de esta búsqueda se obtuvieron 196 referencias. En primer lugar, se realizó un cribado para garantizar que todos los textos se referían al ámbito del deporte y se eliminaron los textos relacionados con experimentación animal Una vez aplicado este filtro y eliminadas las entradas repetidas, el número total de referencias relacionadas con estos conceptos fueron 110 . De estas 110 referencias, una de ellas era un libro, 10 eran capítulos de libro, otro documento eran las actas de un congreso y las 98 entradas restantes eran artículos publicados en revistas científicas. Tan solo dos de estos artículos fueron escritos por un único autor, en el resto la autoría corresponde a varios autores. Los artículos fueron publicados en un total de 72 revistas científicas de diferentes países como Estados Unidos, Alemania, Francia, España, Croacia, Serbia, Noruega, China, Suiza, Brasil e Italia.

En la línea del trabajo de García-Parra, González y Garcés de los Fayos (2016), en la Figura 1 puede apreciarse la dis- tribución de publicaciones por año, siendo 2012 y 2013 los años en los que más publicaciones se recogen. Destacan en el otro extremo 2011 y 2016 como los años en los que menos textos aparecen sobre esta temática.

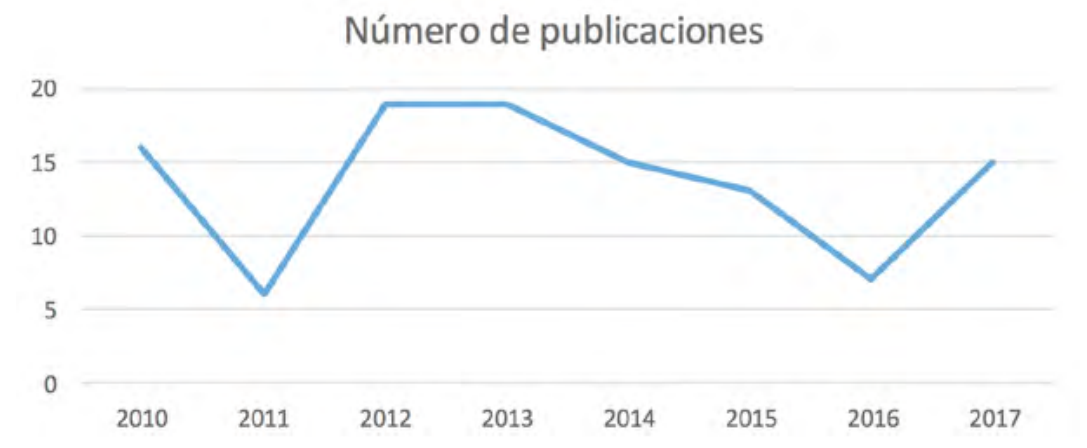

Figura 1. Distribución por años de Número de Publicaciones Relacionadas con Sobrecarga y Sobreentrenamiento en el Ámbito Deportivo.

Las temáticas abordadas por estos artículos también son muy variadas. El 10,9\% de los textos analizados son textos de revisión, el $14.54 \%$ se refieren a validación de instrumentos para la medida del sobreentrenamiento y el $40.9 \%$ de los trabajos abordan este tema desde un planteamiento general y tan solo el 3.6\% se refieren a estudios de caso. El resto de trabajos abordan temáticas muy diversas relacionadas con lesiones como consecuencia del sobreentrenamiento, la afectación del sistema inmunológico, del sistema endocrino, prevención relacionada con pautas nutricionales... entre otras. Las modalidades deportivas estudiadas también son muy dispares: voleibol, fútbol, hockey, natación, rugby, lucha y tenis.

A continuación, en la Tabla 2 se presenta un resumen de los trabajos más representativos, seleccionado los textos que abordan la problemática del sobreentrenamiento y la sobrecarga desde la perspectiva psicológica, en el que emplean fundamentalmente autoinformes para su evaluación.

Tabla 2. Algunas investigaciones relevantes acerca del sobreentrenamiento

\begin{tabular}{|c|c|c|c|c|}
\hline $\begin{array}{l}\text { Le Meur, Buchheit, Au- } \\
\text { bry, Coutts y Hausswirth } \\
(2017)\end{array}$ & $\begin{array}{l}\text { Estudio dela frecuencia } \\
\text { cardiaca máxima ante el } \\
\text { ejercicio físico intenso y } \\
\text { moderado. }\end{array}$ & $\begin{array}{l}20 \text { triatletas varones (8 su- } \\
\text { jetos control y } 13 \text { sujetos } \\
\text { con sobrecarga) }\end{array}$ & $\begin{array}{l}\text { Frecuencia cardíaca } \\
\text { máxima y la valoración del } \\
\text { esfuerzo percibido (RPE) }\end{array}$ & $\begin{array}{l}\text { Cuando se alcanza la frecuen- } \\
\text { cia cardiaca máxima después } \\
\text { del ejercicio moderado, por } \\
\text { ejemplo durante el calen- } \\
\text { tamiento, se considera una } \\
\text { señal de sobreentreamiento. }\end{array}$ \\
\hline
\end{tabular}


Tabla 2. (Continuación)

\begin{tabular}{llll}
\hline \multicolumn{1}{c}{ Autores } & \multicolumn{1}{c}{ Objetivo } & Muestra & Instrumentos de medida \\
\hline Siegl et al. (2017) & $\begin{array}{l}\text { Determinar si una prueba } \\
\text { de carrera submáxima } \\
\text { podría reflejar un estado } \\
\text { de sobrecarga }\end{array}$ & 14 corredores entrenados & $\begin{array}{l}\text { Test de carrera submáxi- } \\
\text { ma de Lamberts, tasa } \\
\text { cardíaca, RPE. }\end{array}$ \\
\hline $\begin{array}{lll}\text { Piacentini y Meeusen } \\
\text { (2014) }\end{array}$ & $\begin{array}{l}\text { Este estudio de caso } \\
\text { longitudinal evaluó la efec- } \\
\text { tividad de un sistema de } \\
\text { monitoreo para prevenir la } \\
\text { sobrecarga no funcional }\end{array}$ & $\begin{array}{l}\text { Estudio de caso: una } \\
\text { atleta femenina de pista y yo } \\
\text { campo }\end{array}$ & $\begin{array}{l}\text { Poms, diario de entre- } \\
\text { namiento y OR score (un } \\
\text { parámetro de medición } \\
\text { específico para detectar } \\
\text { sobrecarga no funcional) }\end{array}$ \\
\hline
\end{tabular}

Mercado y Gallo (2014)

\author{
Determinar la frecuencia \\ de síntomas tempranos \\ del SSE en deportistas de \\ Antioquia en el período \\ precompetitivo antes de \\ los Juegos Nacionales, \\ Colombia 2012
}

\section{3 deportistas de alto} rendimiento participantes en los Juegos Nacionales, Colombia 2012, pertenecientes a 28 disciplinas diferentes
CSSE de la SFMD
Resultados / Conclusiones

El estudio muestra que el Test de carrera Submáximo de Lamberts es capaz de reflejar los primeros síntomas de sobreentrenamiento.

Se observaron puntuaciones más elevadas en OR score y en las escalas de fatiga, depresión y en la puntuación total del POMS como respuesta a estresores personales, sin aumentar las cargas del entrenamiento. Las puntuaciones de la escala de depresión descendieron

Los deportistas afectados por síntomas tempranos del SSE tenían un mayor volumen de entrenamiento en horas por semana y pertenecían a una disciplina individual. También se encontraron mayores estresores ajenos al entrenamiento entre quienes manifestaron síntomas tempranos del SSE.

Aumentar la carga del entre33 triatletas masculinos, 23 de ellos asignados a un grupo de entrenamiento intenso y 10 de ellos rea-

Captación máxima de oxígeno, rendimiento en el ciclismo. namiento puede aumentar el rendimiento cuando aún no se han presentado problemas de sobrecarga, es decir cuando aún los atletas tienen una buena adaptación al entrenamiento. normal

eléficit de rendimien ga funcional

(a)
Solo dos escalas presenta- ron diferencias significativas entre los periodos anterior y posterior a la competición: la escala de Fatiga y la de Éxito. La experiencia acu- mulada en la competición de los atletas senior se vio reflejada en la técnica.

ki, Soares, y de Mello (2014)
El objetivo de este estudio psicológicos del sobreentrenamiento en atletas de élite durante los períodos previos y posteriores a la competición.
48 atletas de judo de un equipo de élite: 24 junior (hasta 18 años) y 24 senior (mayores de 18 años).
RESTQ-Sport antes y después de la competición
Detectar las variables relacionadas con la sobrecarga para evitar la aparición de sobreentrenamiento
24 atletas separados en 2 grupos: entrenamiento intensivo y entrenamiento normal
Variaciones de la frecuencia cardíaca, análisis de sangre, RPE
11 de los 12 de entrenamiento intensivo fueron diagnosticados con sobrecarga. Las medidas más discriminantes fueron la frecuencia cardíaca, el lactato y el RPE.

Monitorizar el rendimiento y los cambios en el estrés-recuperación pueden ser útiles para la detección de la sobrecarga en su etapa más temprana. recuperación de estrés en jugadores de fútbol con sobrecarga

\author{
94 futbolistas
}

Shuttle

STAI, POMS

Variabilidad del ritmo cardíaco, muestreo de sangre 
Tabla 2. (Continuación)

\begin{tabular}{|c|c|c|c|c|}
\hline Autores & Objetivo & Muestra & Instrumentos de medida & Resultados / Conclusiones \\
\hline Tobar (2012) & $\begin{array}{l}\text { Comparar el estado de } \\
\text { ánimo en función del } \\
\text { nivel de ansiedad de los } \\
\text { deportistas }\end{array}$ & $\begin{array}{l}58 \text { nadadores: } 35 \text { hom- } \\
\text { bres, } 23 \text { mujeres }\end{array}$ & $\begin{array}{l}\text { (testorterona, cortisol, } \\
\text { urea sérica), velocidad } \\
\text { explosiva y aptitud cardio- } \\
\text { rrespiratoria, RESTQ-Sport }\end{array}$ & $\begin{array}{l}\text { Los nadadores ansiosos } \\
\text { de baja ansiedad-rasgo } \\
\text { experimentaron mayores } \\
\text { variaciones en los estados } \\
\text { de ánimo durante el periodo } \\
\text { de sobreentrenamiento. }\end{array}$ \\
\hline Schmikli et al. (2011) & $\begin{array}{l}\text { Para estudiar si el test de } \\
\text { campo permite hacer una } \\
\text { distinción válida entre los } \\
\text { atletas con sobrecarga no } \\
\text { funcional y los atletas del } \\
\text { grupo control. }\end{array}$ & $\begin{array}{l}129 \text { atletas de élite } \\
\text { jóvenes, } 77 \text { jugadores de } \\
\text { fútbol y } 52 \text { corredores de } \\
\text { media distancia }\end{array}$ & POMS & $\begin{array}{l}\text { Los atletas que mostraron } \\
\text { un descenso en su rendi- } \\
\text { miento junto con los sínto- } \\
\text { mas típicos del estado de } \\
\text { sobrecarga no funcional: } \\
\text { puntuaciones más eleva- } \\
\text { das en la escala de Cólera } \\
\text { y Depresión del POMS y } \\
\text { una respuesta de cortisol } \\
\text { atenuada. }\end{array}$ \\
\hline Slivka et al. (2010) & $\begin{array}{l}\text { Proponer un incremento } \\
\text { en el rendimiento para } \\
\text { comprobar si aparecían } \\
\text { correlatos con la sintoma- } \\
\text { tología clásica de sobreen- } \\
\text { trenamiento }\end{array}$ & 8 ciclistas varones & $\begin{array}{l}\text { Testosterona y el cortisol } \\
\text { salivales, el rendimiento } \\
\text { en la prueba contrarreloj y } \\
\text { POMS }\end{array}$ & $\begin{array}{l}\text { Al intensificar el rendimiento } \\
\text { durante } 21 \text { días ( } 3.211 \text { km) } \\
\text { la escala de Vigor del POMS } \\
\text { disminuyó desde el día } 1 \\
\text { al día } 4 \text { y permaneció bajo } \\
\text { hasta el final. Sin embargo, } \\
\text { no hubo otros cambios esta- } \\
\text { dísticamente significativos } \\
\text { relacionados con el sobreen- } \\
\text { trenamiento. }\end{array}$ \\
\hline $\begin{array}{l}\text { Dupuy, Renaud, Bherer y } \\
\text { Bosquet (2010) }\end{array}$ & $\begin{array}{l}\text { Investigar si el rendimien- } \\
\text { to cognitivo era un marca- } \\
\text { dor válido de sobrecarga }\end{array}$ & $\begin{array}{l}10 \text { atletas de resistencia } \\
\text { masculinos }\end{array}$ & $\begin{array}{l}\text { Stroop, prueba de veloci- } \\
\text { dad constante }\end{array}$ & $\begin{array}{l}\text { Al aumentar la carga de } \\
\text { entrenamiento en un 100\% } \\
\text { durante } 2 \text { semanas, } 5 \text { atletas } \\
\text { mostraron sobrecarga. Esto } \\
\text { se acompañó de deterioro de } \\
\text { algunas funciones ejecu- } \\
\text { tivas, en este caso con un } \\
\text { incremento del tiempo de } \\
\text { reacción en la prueba Stroop }\end{array}$ \\
\hline
\end{tabular}




\section{Discusión}

El sobreentrenamiento está compuesto por varios síntomas, el cual puede considerarse como un síndrome (González-Boto, Andrade y Márquez, 2006); Lemyre et al., 2007; Mora, 2006; Pancorbo, 2003; Subiela y Subiela, 2011). Destacar cambios a nivel general, orgánicos, cognitivos, emocionales, conductuales y sociales que afectan a la salud y el rendimiento del deportista. A pesar de ello, en ocasiones el descenso del rendimiento es el indicador de sobreentrenamiento más visible y llamativo para el observador externo, aunque es posible que el deportista lleve tiempo manifestando alteraciones y que no son detectadas a simple vista, ya que al comienzo la sintomatología que se presenta suele ser difusa (Dupuy et al., 2010; Nicholls et al., 2011; Sánchez-Beleña, 2011).

Destacar que casi $1 / 3$ de los deportistas de alto rendimiento han padecido alguna vez sintomatología asociada al sobreentrenamiento (Subiela y Subiela, 2011). La aparición del sobreentrenamiento suele surgir cuando hay un aumento importante de la carga de trabajo, intensidad de los ejercicios y horas de entrenamiento a la semana, así como un insuficiente tiempo de recuperación entre las sesiones de entrenamiento (Dupuy et al., 2010; Mercado y GaIlo, 2014; Nicholls et al., 2011), siendo perjudicial el aumento de trabajo cuando hay algún tipo de síntoma al respecto (Aubry et al., 2014). Además, la falta o malos hábitos de descanso y sueño, alimentación y ocio, facilitan el sobreentrenamiento y el retraso de la recuperación (Molinero et al., 2011). Y por último, es necesario atender a los estresores psicosociales o externos al entrenamiento, que en algunos de los estudios analizados, también estaban relacionados con la aparición del sobreentrenamiento (Subiela y Subiela, 2011; Mercado y Gallo, 2014; Piacentini y Meusen, 2014).

Por su parte, el psicólogo del deporte (Cantón, 2016) puede asesorar y orientar a los técnicos deportivos (p. ej., entrenadores y preparadores físicos) en materia de sobreentrenamiento y sus efectos negativos desde una perspectiva psicológica, así como colaborar en el desarrollo de un plan de acción interdisciplinar orientado a la mejora global del rendimiento y la salud del deportista (p. ej., orgánica, física y psicológica). Los técnicos deportivos pueden modular o adaptar sus entrenamientos en función de la intensidad de los ejercicios, el tiempo de entrenamiento, la carga de trabajo y los tiempos de recuperación entre sesiones. Además, cuando se requiera, pueden organizar ejercicios variados y entretenidos que beneficien el disfrute y la distensión del deportista. Por último, supervisar y aconsejar al deportista sobre los hábitos saludables de descanso y sueño, alimentación y ocio.
A nivel de intervención indirecta, el psicólogo del deporte puede trabajar con los técnicos deportivos para que éstos potencien sus entrenamientos a nivel psicológico. Por ejemplo, ayudarle a establecer objetivos psicológicos para cada entrenamiento (motivación, disfrute, autoconfianza, reducir carga mental, etc.) y mejorar el liderazgo y gestión del deportista (aplicación del refuerzo positivo o castigo negativo, comunicación positiva, etc.).

En cuanto a la intervención directa, el psicólogo del deporte tiene que realizar un proceso de evaluación (como se verá más adelante) e intervención psicológica (p. ej., técnicas de control estimular, estrategias de autorregulación emocional y afrontamiento), tanto con el deportista como con su entorno y estilo de vida. Y por último, otra cuestión importante a trabajar con los deportistas, conjuntamente con los servicios médicos (p. ej., médicos, fisioterapeutas y nutricionistas), son los hábitos de descanso y sueño, alimentación, ocio y recuperación de lesiones.

Por todo ello, es importante la detección temprana y seguimiento de los posibles indicadores de sobreentrenamiento en el ámbito deportivo, el cual habitualmente ha sido evaluado a través de medidas biológicas (Meeusen et al., 2006), autoinformes (Molinero et al., 2011), o bien combinando ambas medidas (Clemente et al., 2009).

Desde una perspectiva psicológica, las variaciones del estado de ánimo aparecen en los primeros momentos del desarrollo del sobreentrenamiento y el POMS es uno de los instrumentos más empleado y eficaces para detectarlo, observándose un descenso del vigor y un aumento de la cólera, fatiga y depresión cuando se padece (Ackel-D’Elia et al., 2010; Andrade, Arce y Seoane, 2000; Molinero et al., 2011; Piacenti y Meeusen, 2014; Schmikli et al., 2011; Slivka et al., 2010). Otros autores añaden la necesidad de evaluar también los niveles de ansiedad y estrés de los deportistas, variables relacionadas con cambios emocionales (Brink et al., 2012; Tobar, 2012), así como detectar posibles situaciones estresantes de la vida deportiva o extradeportiva del mismo (Anshel, 2003; Pancorbo, 2003; Prendas et al., 2007).

Destacar que las versiones reducidas del POMS (Andrade et al., 2013; Andrade et al., 2011) lo convierte en un instrumento de fácil y rápida aplicación, incluso para tomar medidas repetidas en diferentes momentos del entrenamiento, la competición o temporada, y así monitorizar las variaciones del estado de ánimo que podrían ser las primeras señales del sobreentrenamiento.

Por su parte, el esfuerzo percibido (Le Meur et al., 2013; Le Meur et al., 2017; Siegl et al., 2017) y el nivel de fatiga (Noce et al., 2014; Ten Haaf et al., 2017) se asocian positivamente con el sobreentrenamiento, mientras que la motivación al 
éxito (Noce et al., 2014) y la predisposición a entrenar (Ten Haaf et al., 2017) disminuyen cuando se padece.

Dentro de las medidas fisiológicas analizadas, cabe destacar la frecuencia cardíaca, que también resulta importante en los primeros momentos de los estados de fatiga. Sobre todo se observa como problemático su incremento en estado de reposo y ante el ejercicio físico moderado (Le Meur et al., 2013; LeMeur et al., 2017). Los técnicos deportivos, de forma sencilla, podrían registrar la frecuencia cardíaca de los deportistas antes de los entrenamientos, al inicio de la semana o en determinados momentos que se detecten como críticos (p. ej., inicio o final de la temporada).

Como se puede intuir, una vez más el psicólogo del deporte (Cantón, 2016) tiene un rol fundamental en la evaluación del sobreentrenamiento, por ejemplo, a través de la medición de los estados de ánimo, esfuerzo percibido y fatiga. Esto permite conocer las variaciones y cómo está respondiendo cada deportista (monitoreo individual) en los diferentes momentos de la temporada (p. ej., durante la pre temporada, durante o final de la misma) o situaciones puntuales de la misma (p.ej., al inicio de una semana tras un alto esfuerzo de entrenamiento o después de una competición importante de mucho desgaste físico y emocional). Para ello es importante sensibilizar a todos los estamentos del ámbito deportivo (directores deportivos, entrenadores, preparadores físicos, médicos, fisioterapeutas, nutricionistas, psicólogos y familiares), para que trabajen al unísono, fomentando el autoconocimiento del deportista, siendo más sensibles a las variaciones individuales y pidiendo ayuda cuando se requiera.

Por su parte, Brink et al. (2012) señala la importancia de monitorizar también el rendimiento deportivo, por lo que la evaluación del mismo puede ser un indicador no solo de su valía deportiva, si no también, de un posible bajo rendimiento debido al sobreentrenamiento. Esta labor podría quedar en manos de los técnicos deportivos, con la colaboración del psicólogo del deporte, el cual le puede ayudar en la definición conductual y/o categorías a registrar, establecer un instrumento de registro, entrenarles en observación, realizar el análisis de los datos e interpretar los resultados.

En relación al rendimiento deportivo, en ocasiones se busca un incremento de la carga de entrenamiento para corregir un descenso del mismo. El problema surge cuando esta disminución es un síntoma del sobreentrenamiento, y en este caso, el organismo responderá mal al intensificar el trabajo, aumentando el riesgo de mala adaptación al entrenamiento y con ello precipitando la aparición de sobreentrenamiento (Aubry et al., 2014).

Una vez que las manifestaciones de sobreentrenamiento ya se han presentado, entonces se debe interrumpir la actividad deportiva e intervenir sobre la sintomatología específi- ca desde una perspectiva interdisciplinar, pero la prevención sigue siendo el mejor tratamiento. Por ello sería interesante desarrollar programas de prevención del sobreentrenamiento, en el que un elemento básico sería la psicoeducación, conociendo lo que son los estados de ánimo y su identificación para hacer conscientes a los deportistas de las posibles variaciones vinculadas al sobreentrenamiento.

\section{Referencias}

Ackel-D’Elia, C., Vancini, R. L., Castelo, A., Nouailhetas, V. L. A. y da Silva, A. C. (2010). Absence of the predisposing factors and signs and symptoms usually associated with overreaching and overtraining in physical fitness centers. Clinics 65, 1161-1166. http://dx.doi.org/10.1590/S1807-59322010001100019

Andrade, E., Arce, C., De Francisco, C., Torrado, J. y Garrido, J. (2013). Versión breve en español del cuestionario POMS para deportistas adultos y población general. Revista de Psicología del Deporte, 22, 95-102

Andrade, E., Arce, C., Garrido, J., Torrado, J. y De Francisco, C. (2011). Modelo de medida del estado de ánimo subjetivo en deportistas adolescentes. Revista de Psicología del Deporte, 20, 537-548.

Andrade, E. M., Arce, C. y Seoane, G. (2000). Aportaciones del POMS a la medida del estado de ánimo de los deportistas: estado de la cuestión. Revista de Psicología del Deporte, 9(1-2), 7-20.

Andrade, E. M., Arce, C. y Seoane, G. (2002). Adaptación al español del cuestionario "Perfil de los Estados de Ánimo" en una muestra de deportistas. Psicothema, 14, 708-713.

Andrade, E., España, S. y Rodríguez, D. (2016). Factores de estado de ánimo precompetitivo en adolescentes de deportes colectivos y satisfacción con el rendimiento. Revista de Psicología del Deporte, 25, 229-236.

Anshel, M., H. (2003). Sport Psychology: from theory to practice. San Francisco, Estados Unidos: Benjamin Cummings.

Aubry, A., Hausswirth, C., Louis, J., Coutts, A. J. y Le Meur, Y. (2014). Functional overreaching: the key to peak performance during the taper? Medicine and Sciences of Sports and Exercise, 46, 1769-1777.

Bonete, E., Moya, M. y Suay, F. (2009). La escala de confusión del POMS como indicador del impacto de la carga del entrenamiento. Revista Iberoamericana de Psicología del Ejercicio y el Deporte, 4, 289-304.

Borg, G. (1970). Perceived exertion: a note on "history and methods". Medicine and Science of Sports and Exercise, 5, 90-93.

Brink, M. S., Visscher, C. Coutts, A. J. y Lemmink, K. A. (2012). Changes in perceived stress and recovery in overreached young elite soccer players. Scandiavian Journal of Medicine and Science in Sports, 22(2), 285-292. https://10.1111/j.1600$0838.2010 .01237 . x$

Budgett, R. Newsholme, E., Lehmann, M., Sharp, C., Jones, D., Jones, T., White P. (2000). Redefining the overtraining syndrome as the unexplained underperformances syndrome. British Journal of Sports Medicine, 3(1), 67-68. http://dx.doi.org/10.1136/ bjsm.34.1.67

Revista de Psicología Aplicada al Deporte y al Ejercicio Físico (2017), 2, e12, 1-12 
Cadegiani F. A. y Kater, C. (2017). Hormonal aspects of overtraining syndrome: a systematic review. BMC Sports Science, Medicine and Rehabilitation, 9, 14. https://doi.org/10.1186/s13102017-0079-8.

Cantón, E. (2016). La especialidad profesional en Psicología del Deporte. Revista de Psicología Aplicada al Deporte y al Ejercicio Físico, 1, e2. https://doi.org/10.5093/rpadef2016a2

Carlín, M. y Garcés de los Fayos (2010).El síndrome de burnout: Evolución histórica desde el contexto laboral al ámbito deportivo. Anales de Psicología, 26(1), 169-180.

Clemente, V., Muñoz, V., Ramos, D., Navarro, F., y González Ravé, J. M. (2009) Muscle destruction, heart rate, lactate and perceived exertion in an ultra-endurance $24 \mathrm{~h}$ relay race. Motricidad. European Journal of Human Movement, 24, 121

De la Vega, R., Galán, A., Ruiz-Barquín, R. y Tejero, C. (2013). Estado de ánimo precompetitivo y rendimiento percibido en Boccia paralímpica. Revista de Psicología del Deporte, 22, 39-45.

De la Vega R., Ruiz-Barquín, R., Borges, P. J. y Tejero-González, C.M. (2014). Una nueva medida tridimensional del estado de ánimo deportivo: el POMS-VIC. Cuadernos de Psicología del Deporte, 14(2), 37-46.

De la Vega, R., Ruiz-Barquín, R., Tejero, C. y Rivera, M. (2014). Relación entre estados de ánimo y rendimiento en voleibol masculino de alto nivel. Revista de Psicología del Deporte, 23, 49-56.

Del Villar, A., Pegueros, A. y Franco, G. (2008). Questionnaire for de-tection of early signs of overtraining: adjustment and evaluation in mexican athletes. Archivos de Medicina del Deporte, $25,573$.

Dupuy, O., Renaud, M., Bherer, LyBosquet L. (2010). Effect of functional overreaching on executive functions. International Journal of Sports Medicine, 37, 617-623. https://10.1055/s-0030-1255029

Freudenberger, H. J. (1974). Staff burnout. The Journal of Social Issues, 30(1), 159- 166.

García-Naveira, A. y Locatelli, L. (2015). Psychological benefits in Physical Activity and sports. En R. Wolfe (Ed.), Psychological health and needs research developments (pp. 91-103). Hauppauge NY, Estados Unidos: Nova Science Publisher.

García-Parra, N., González, J. y Garcés de los Fayos E. J. (2016). Estado actual del estudio del síndrome de burnout en el deporte. Cuadernos de Psicología del Deporte, 16(2), 21-28.

González-Boto, A., Andrade, A. y Márquez, S. (2006). La adaptación deportiva y el sobreentrenamiento: mecanismos e hipótesis explicativas. Revista de Entrenamiento Deportivo, 20(2), 21- 31.

González-Boto R., Molinero, O. y Márquez, S. (2006). El sobrentrenamiento en el deporte de competición: implicaciones psicológicas del desequilibrio entre estrés y recuperación. Ansiedad y estrés, 12(1), 99-115.

González-Boto, R., Salguero, A., Tuero, C., Kellmann, M. y Márquez, S. (2008). Spanish adaptation and analysis by structural equation modeling of an instrument for monitoring overtraining: The Stress-Recovery Questionnaire (RESTQ-Sport). Social Behavior and Personality, 36, 635-650. https://doi.org/10.2224/ sbp. 2008.36.5.635

Kellman, M. (2002). Underecoveringand overtraining: different concepts - similar impact? En M. Kellman (Ed.), Enhancing recovery: preventing underperformance in athletes (pp. 3-24). Champaign, IL, Estados Unidos: Human Kinetics.
Kellman, M. y Kallus, K. (2001). Recovery-Stress Questionnaire for Athletes. User Manual. Champaign, IL, Estados Unidos: Human Kinetics.

Le Meur, Y, Buchheit, M, Aubry, A, Coutts, A. J y Hausswirth, C. (2017). Assessing Overreaching With Heart-Rate Recovery: What Is the Minimal Exercise Intensity Required? International Journal of Sports Physiology Performance, 12, 569-573. https://doi.org/70.1723/iispp. 2015-0675

Le Meur, Y., Hausswirth, C., Natta, F., Couturier, A., Bignet, F. y Vidal, P. P. (2013). A multidisciplinary approach to overreaching detection in endurance trained athletes. Journal of Applied Physiology, 174, 411-420.

Legros, P. (1993). Le surentraînement. Science and Sports, 7, 51-57.

Lemyre P. N., Roberts, G. C. y Stray-Gundersen, J. (2007). Motivation, overtraining and burnout: can self- determined motivation predict overtraining and burnout in elite athletes? European Journal of Sport and Science, 7(2), 115-126. https://doi. org/10.1080/17461390701302607

LeUnes, A. y Burger, J. (2000). Profile of mood states research in sport and exercise psychology: Past, present and future. Journal of Applied Sport Psychology, 12, 5-15. https://doi. org/10.1080/10413200008404210

Márquez, S. (2006). Estrategias de afrontamiento del estrés en el ámbito deportivo: fundamentos teóricos e instrumentos de evaluación. International Journal of Clinical and Health Psychology, 6, 359-378.

Maso, F., Lac, G. y Brun, J. F. (2005). Analysis and interpretation of SFMS questionnaire for the detection of early signs of overtraining: a multicentric study. Science and Sports, 20, 12-20. https:// doi.org/10.1016/i.scispo.2004.05.013

Martin, D. T., Andersen, M. B. y Gates, W. (2000). Using Profile of Mood States (POMS) to monitor high-intensity training in cyclists: Group versus case studies. The Sport Psychologist, 14, 138-156.

McNair, D.M., Lorr y M. Doppleman, L.F (1971). Profile of Mood States Manual. San Diego, Estados Unidos: Educational and Industrial Testing Service.

Meeusen, R. (1999). Overtraining and the central nervous system, the missing link? En M. Lehman, C. Foster, U. Gastman, H. Keizer y J. Steinacker (Eds.), Overload, performance incompetence and regeneration in sport (pp. 187-202). New York, Estados Unidos: K/uwer Academic/Plenum Publishers.

Meeusen, R., Duclos, M., Gleeson, M., Rietjens, G., Steinacker, J. y Urhausen, A. (2006). Prevention, diagnosis and treatment of the overtraining syndrome. European Journal of Sport Science, 6(1), 1-14. https://doi.org/10.1080/17461390600617717

Meeusen, R., Piacentini, M. Busschaert, B., Buyse, L., De Schutter, R. y Stray-Gundersen, J. (2004). Hormonal responses in athletes: the use of a two bout exercise protocol to detect subtle differences in (over)training status. European Journal of Appied Pshysiology, 91, 140-146. https://doi.org/10.1007/s00421-003-0940-1

Mercado, M. A. y Gallo J. A. (2014). Manifestaciones tempranas de sobreentrenamiento en deportistas en el período precompetitivo antes de unos juegos nacionales: un estudio transversal. Latreia, 27, 375-385.

Molinero, O., Salguero, A. y Márquez, S. (2011). Análisis de la recuperación-estrés en deportistas y relación con los estados de ánimo: Un estudio descriptivo. Cuadernos de Psicología del Deporte, 17(2), 47-55. 
Mora, M. (2006). Identificación de sintomas de sobreentrenamiento y burnout en futbolistas hombres costarricenses de segunda división en función de los estados anímicos, apoyo percibido de figuras significativas, edad de inicio, experiencia competitiva y desempeño colectivo. Tesis doctoral. Universidad Nacional de Heredia, Costa Rica.

Nicholls, A. R., McKenna, J., Polman, R. C. J. y Backhouse, S. H. (2011). Overtraining during preseason: Stress and negative affective states among professional rugby union players. Journal of Clinical Sport Psychology, 5, 211-222. https://doi. org/10.1123/jesp.5.3.211

Noce, F., Costa, V. T., Szmuchrowski, L. A., Soares, D. S. y de Mello, M. T. (2014). Psychological indicators of overtraining in high level judo athletes in pre- and post-competition periods. Archives of Budo, 10, 245-251.

Nogueira, A., Salguero, A y Márquez, S. (2017). Adicción a correr: una revisión desde sus inicios hasta la actualidad. Revista de Psicología Aplicada al Deporte y al Ejercicio Físico, 1, e6. https:/l doi.org/10.5093/rpadef2017a1

Pancorbo, A. E. (2003). Diagnóstivo y prevención de la fatiga crónica o del síndrome de sobreentrenamiento en el deporte de alto rendimiento. Una propuesta de mecanismos de recuperación biológica. Cuadernos de Psicología del Deporte, 3 (1), 61 - 80.

Piacentini, M. F. y Meeusen, R. (2014). An online training-monitoring system to prevent nonfunctional overreaching. International Journal of Sports Physiology Performance, 10, 524-527. https://doi.org/10.1123/ijspp.2014-0270

Prendas, J. L., Castro, W. y Araya-Vargas, G. (2007). Identificación y comparación de síntomas de desgaste físico y mental en árbitros, entrenadores y educadores físicos. Revista de Ciencias del Ejercicio Físico y de la Salud, 5(1), 55-69.

Sánchez-Beleña, F. (2011). Análisis de las variables relacionadas con el sobreentrenamiento y la salud de las bailarinas. (Tesis Doctoral). Facultad de Psicología. Universidad Complutense de Madrid.

Saremi, J. (2009). Overtraining Syndrome. American Fitness, 27(1), 10-15.

Schmikli, S. L., Brink, M. S., De Vries, W. R., Backx, F. J. G (2011). Can we detect non-functional overreaching in young elite soccer players and middle-long distance runners using field performance tests? British Journal of Sports Medicine, 45, 631-636.

Selye, H. (1936). A syndrome produced by diverse nocuous agents. Nature, 138, 32

Siegl, A., M Kösel, E., Tam N., Koschnick, S., Langerak, N. G., Skorski, S., Meyer, T. y Lamberts, R. (2017). Submaximal markers of fatigue and overreaching; implications for monitoring athle- tes. International Sport Medicine, 38, 675-682. https://doi.org/10.1055/s-0043-170226

Slivka, D. R., Hailes, W. S., Cuddy, J. S. y Ruby, B. C. (2010). Effects of 21 days of intensified training on markers of overtraining. Journal of Strength and Conditioning Research, 24, 2604-2612. DOI: https://doi.org/10.1519/JSC.0b013e3181e8a4eb

Steinacker, J., Lormes, W., Liu, Y. Optiz-Gress, A., Baller, B. Günter, K., ... Altenburg, D. (2000). Training of junior rowers before world Championship. Effects on performance, mood state and selected hormonal and metabolic responses. Journal of Physical Fitness Sport Medicine, 40, 327-335.

Steinacker, J., Lormes, W., Reissnecker, S. y Liu, Y. (2004). New aspects of the hormone and cytokine response to training. European Journal of Applied Physiology, 97, 382-393.

Stevenson, R. (2008). Overtraining in Endurance Athletes: Signs, Symptoms, and Prevention. Competitors in Southern California, 22, 18-19.

Suay, F. y Fernández de Castro, M. (2001). Evaluación del imparto psicobiológico del entrenamiento en atletas de élite. Actas del VIII Congreso Nacional de Psicología de la Actividad Física y el Deporte. Pontevedra, España.

Suay, F., Ricarte, J. y Salvador, A. (1998). Indicadores psicológicos de sobreentrenamiento y agotamiento. Revista de Psicología del Deporte, 13, 7-25.

Suay, F., Sanchís, C y Salvador, A. (1997). Marcadores hormonales de sobreentrenamiento. Revista de Psicología del Deporte, 11, 21-39.

Subiela, J. V. y Subiela J. D. (2011). El síndrome de sobreentrenamiento: criterios diagnósticos y conductas terapéuticas. Academia Biomédica Digital, 48, 1-10.

Ten Haaf, T., Van Staveren, S., Oudenhoven, E., Piacentini, M. F., Meeusen, R., Roelands, B., ... De Koning, J. J. (2017). Prediction of functional overreaching from subjective fatigue and readiness to train after only 3 days of cycling. International Journal of Sports Physiology Performance 12, 287-294. https://doi. org/10.1123/ijspp.2016-0404

Tobar, D. A. (2012). Trait anxiety and mood state responses to overtraining in men and women college swimmers. International Journal of Sport and Exercise Psychology, 10, 135-148. https://doi.org/10.1080/1612197X.2012.666399

Yoke, M. (2006). Personal fitness training theory and practice. California: Sherman Oaks. Aerobics and Fitness Association of America.

Zintl, F. (1991). Entrenamiento de la resistencia. Barcelona, España: Martínez Roca. 